\title{
Analysis of single-operation cold forging of a hollow ball from a tubular billet
}

\author{
Grzegorz Samołyk $^{1} \cdot$ Grzegorz Winiarski $^{1}$
}

Received: 3 December 2018 / Accepted: 15 April 2019 /Published online: 2 May 2019

(C) The Author(s) 2019

\begin{abstract}
The article reports the results of an experimental and theoretical study of a single-stage cold forging process. This process was used to produce hollow balls with an outside diameter of $30 \mathrm{~mm}$, with two central openings. The forgings were made from tubular aluminum alloy billets with wall thicknesses of $3 \mathrm{~mm}$ and $4 \mathrm{~mm}$. The other dimensions of the billets (outer diameter and height) were varied, always making sure that the volume of the forging remained constant. The results of the study were discussed with a special focus on forming conditions, quality of the forging, potential limitations of the process, and the impact of billet dimensions (also expressed as non-dimensional slenderness ratios) on the conditions of the forming process.
\end{abstract}

Keywords Hollow ball $\cdot$ Die forging $\cdot$ Cold forging $\cdot$ AlMgSi0.5 alloy

\section{Introduction}

The ball, as a structural and functional element, is one of the basic parts widely used in the construction of machines, structures, etc. Balls are produced from various materials, not only metals but also polymers, ceramics, and composites [1]. The application of balls is also determined by their size, which can range from the microscale to the order of several dozen millimeters. In addition, balls can be solid or hollow [2, 3]. All these different types of balls are manufactured using different technologies. The choice of a technology also depends on the production volume as determined by the demand for the particular type of product (ball). In the case of balls made of metal alloys, methods such as metal forming (e.g., rolling, forging, or processes derived from them) or machining are usually used [3-5]. Occasionally, casting methods are also employed. Metal forming is particularly recommended for mass manufacture of products such as rolling bearing balls, balls used in industrial ball mills, or ball valve components. In this last case,

Grzegorz Samołyk

g.samolyk@pollub.pl

Grzegorz Winiarski

g.winiarski@pollub.pl

1 Lublin University of Technology, Mechanical Engineering Faculty, Lublin, Poland the ball forgings are hollow and usually require further shaping by machining $[3,4,6]$. In some cases (usually for economic reasons), such products are made using casting methods; however, compared with forging methods, the products obtained have weaker strength parameters. It is worth mentioning that balls, both solid and hollow, are also used as decorative or construction elements. In those cases, the most attractive solutions are simple and reliable technologies that allow to implement a number of design ideas at a minimal cost [3]. One proposal for such design applications is the cold forging of hollow balls, which is the subject of this paper. Of course, this technology can also be applied in the production of other typical machine parts which have the shape of a hollow ball.

Before we discuss the results of the study, it is worth presenting the genesis of the technology proposed by the authors. A vast majority of known technologies for the production of balls are dedicated to solid products. According to Pater et al. [7], hot forming methods predominate, and the most popular technologies are based on rolling, open die forging, and closed die forging, in which waste material, called flash, is generated. Such solutions are usually used in the production of balls of relatively small diameters, intended for use in rolling bearings. However, in the case of large-sized balls, the preferred methods are those in which no flash is formed.

Due to the growing requirements of production plants and the large demand for large-sized balls, intended for example for grinding mills, forging methods are being replaced by 
rolling methods. The expectations of the industry determine the directions of scientific research, as a result of which, a number of new varieties of rolling methods for ball-type products have been developed over the recent years. Extensive research and development work in this area is carried out, among others, at the Lublin University of Technology. For example, Pater and Tomczak [8] have shown that balls with a diameter of over $40 \mathrm{~mm}$ can be efficiently produced by helical rolling of sections of a scrapped railway rail used as billets. The advantages and disadvantages of helical rolling are discussed in detail in a paper by Tomczak et al. [4], which presents the results of experimental and theoretical investigations and the principles of tool design. In another study, Pater [9] has demonstrated that an equally effective ball-forming process is cross-wedge rolling, in which balls with a diameter below $40 \mathrm{~mm}$ can be formed directly from a billet, e.g., a scrap railhead. As a side note, it is worth mentioning that this technology can also be used to manufacture hollow ball forgings. Pater et al. [5] have also shown that cross-wedge rolling of balls with a diameter of over $60 \mathrm{~mm}$ should be preceded by a step in which the billet is shaped into a rod. A much better solution, however, is to pre-forge ball blanks, for example, by semi-open die forging, as suggested in an earlier study by Tomczak et al. [4, 7]. The semi-finished products thus prepared are then calibrated by helical rolling.

Unfortunately, the known methods of forging or rolling of solid balls are generally not intended for cold forming, and if balls are cold-formed, it is only when they have a relatively small diameter and are made of soft materials. Such a conclusion can be drawn from the results of numerical calculations made by Cao et al. [10]. Those authors have shown that during cold helical rolling, the material is exposed to low-cycle fatigue, which can lead to the formation of internal cracks. This is confirmed by the results of investigations of cold rolling carried out by Pater and Tomczak [8] as well as Samołyk's [11] study of the conditions of cold forming using incremental methods, in which continual changes in the character of the deformation were observed in the cross-section of the material being formed.

Hollow balls are mainly manufactured using impression die forging methods, and rolling methods are used sporadically. Most of the methods used in practice have been developed for making forgings (semi-finished products) or finished parts for use in ball valves. Typical shapes of such hollow ball forgings are shown in Fig. 1.

A characteristic feature of the forgings of ball valve parts is that they have a central hole with a constant cross-section and a relatively large diameter, which is often close to the outer diameter of the ball. Another feature is the characteristic thickness and shape of the wall, which necessitates the use of relatively thin-walled tubular billets for the production of these parts. According to the results obtained by Alhussainy et al. [6], a thin-walled tube subjected to compression, for instance, during forging, is exposed to local or global buckling. This considerably limits the technological possibilities of forging from tubular billets, which usually requires the use of multistage forming methods. One example is a forging method developed by Winiarski [12] for forming ball valve forgings, in which the product is manufactured in two operations, using two sets of tools with an additional mandrel (Fig. 1b). If no mandrel is used, the billet is subject to local buckling. Of course, there is a well-known method of forging ball valves in a single forging operation described by Kwan [13]. Unfortunately, when Kwan's method was used to forge walled products (Fig. 1c), the height of the billet had to be reduced, and the resulting hole had a diameter that was only slightly smaller than the outer diameter of the ball. In order to eliminate the constraints connected with the use of tubular billets, ball forgings can be forged from solid billets. Such a technology was used in a study by Kozjek et al. [14], in which holes were pierced in ball forgings (Fig. 1a). Unfortunately, the holes were blind, and the bottom had to be cut out. In addition, Kozjek et al. [14] demonstrated that this technology significantly reduced tool life and was more energy-intensive.

An alternative process for forging hollow balls is mandrel rolling, as proposed by Kang and Lee [15]. This technology is a variant of the well-known ring rolling process, and the finished product has a shape shown in Fig. 1b. Skrochocki and Tomczak [16] proposed a rotary compression process, in which hollow balls are produced from a tubular billet in multiple operations (Fig. 1d). To obtain the finished forging, the semi-finished blank must be cut and calibrated. This alternative to forging is worth considering when process efficiency is a priority (e.g., in mass production). However, according to Tomczak [17], in this technology, a strong relation between billet dimensions and distribution of thickness of a wall of forging is an essential problem. And so, it is possible to ask questions, whether indeed is it worthwhile replacing the forging process with the other alternative technology? After all, according to Berviller et al. [18] and Chen [19], the forging process is characterized by very much favorable conditions of metal shaping, and the final part has got good mechanical properties and correct layout of fibers. Production costs are comparatively small (i.e., material and tool), and the productivity of the forging process is very large $[18,20]$.

Returning to the theme of paper, hollow ball forgings characterized by a relatively constant wall thickness can be made from tubular billets using die forging. Three forming methods can be distinguished here, all of which are used in the manufacture of general-purpose products. They are shown in Fig. 2.

Especially noteworthy are the technologies developed at the beginning of the second half of the twentieth century. According to a solution proposed by Eklund and Campbell [21], a blank, e.g., an ellipsoid, is formed in the first operation, which is then calibrated to the final shape in the second operation (Fig. 2b), and finished during finishing operations. The 


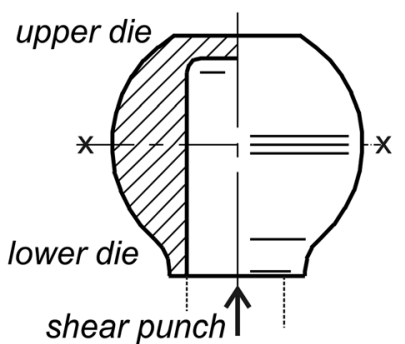

(a)

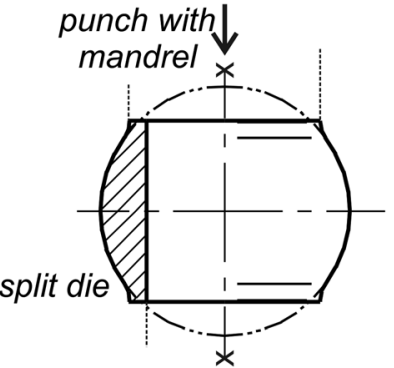

(b)

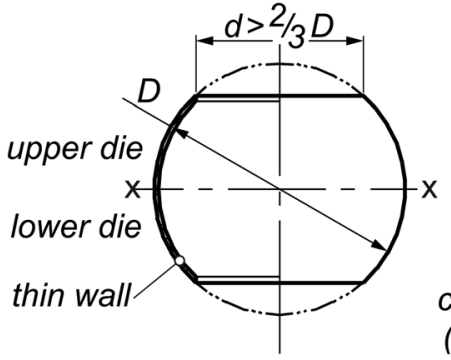

(c)

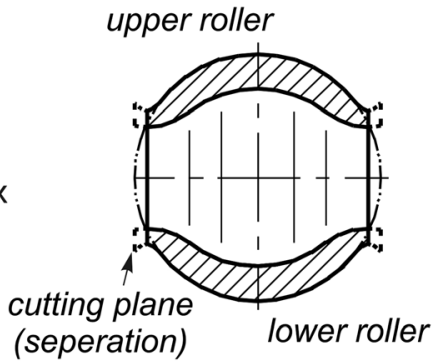

(d)

Fig. 1 Typical shapes of hollow ball forgings intended for use in ball valves and schematics of the forming methods; full description in text

same sort of blank can be obtained in a single operation using a variant of Eklund and Campbell's process [22], which, however, requires additional rotation of the dies (Fig. 2a). Due to technical limitations and insufficient theoretical knowledge, this technology is rarely used in industrial settings. The characteristic feature of the hollow ball forgings produced using this method is the discontinuity of the material, which usually takes the form of central holes (these are the so-called unclosed balls). A solution to this problem has been proposed, among others, by Braginsky and Dashevsky [23], who developed a process for producing closed hollow ball forgings by extruding two ball halves (hemispheric units) and then joining them by friction welding (Fig. 2c).

Summing up, it should be noted that, unfortunately, neither Kwan [13] nor Eklund and Campbell [22] presented in their papers comprehensive studies into the conditions of the forming processes they described or perspectives for further development of the proposed methods, which, let us recall, allow to obtain a general-purpose hollow ball. We treated this as an incentive to conduct the research reported in this present paper. It should also be mentioned that the forming method proposed here shares many common features with the already known solutions, but is a new approach to the technological problem formulated, among others, in an earlier work by Samołyk [2]. Our overriding goal was to develop a method that would allow to obtain ball forgings of any dimension in a single operation in cold forming conditions from thick-walled tubular billets. In addition, we assumed that the forging method should be maximally energy efficient, and the tools should have a simple structure and be suitable for mounting on universal, commonly used forging machines.

\subsection{Characterization of the forging process}

The focus of this paper is the process of forging a hollow ball. A schematic diagram of the process is shown in Fig. 3a (initial phase) and Fig. $3 \mathrm{~b}$ (final phase). In this technology, a tubular billet with an outside diameter $d_{0}$, height $h_{0}$, and initial wall thickness $g_{0}$ is placed in the bottom die. It is assumed that in industrial conditions, the billet is guided into position by a feeding-centering device (gripper) mounted on a robotic arm. The gripper, in addition to feeding and positioning the billet, can also serve as a billet support at the initial stage of forming to improve the stability of the process. After a specified forming time, the gripper is retracted in a preprogrammed direction $x_{1}$ and $x_{2}$. Based on numerical

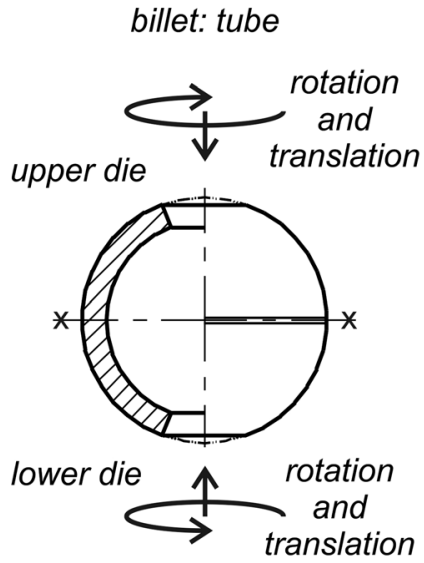

(a)

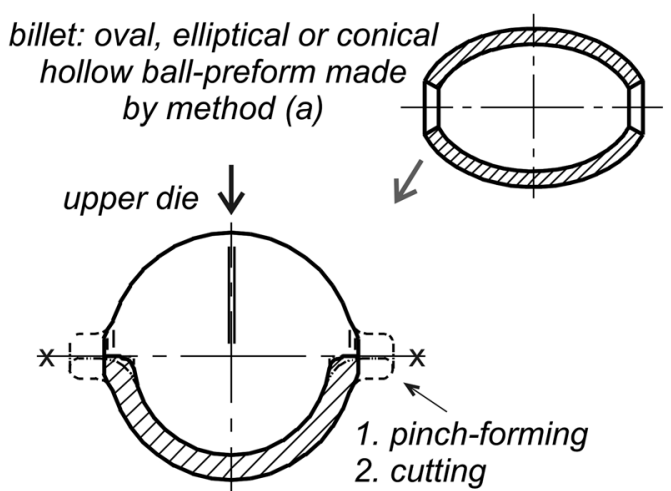

lower die

(b) billet: two hemispheric preforms

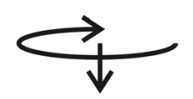

hemispheric unit

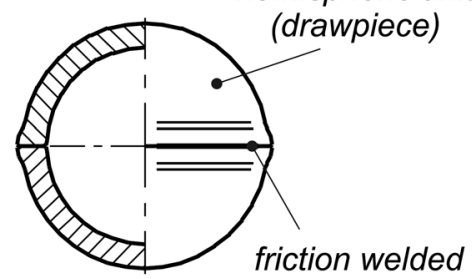

joint

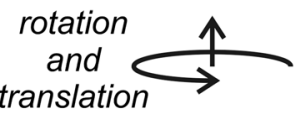

(c)

Fig. 2 Three methods of producing general-purpose hollow ball forgings; description in text 


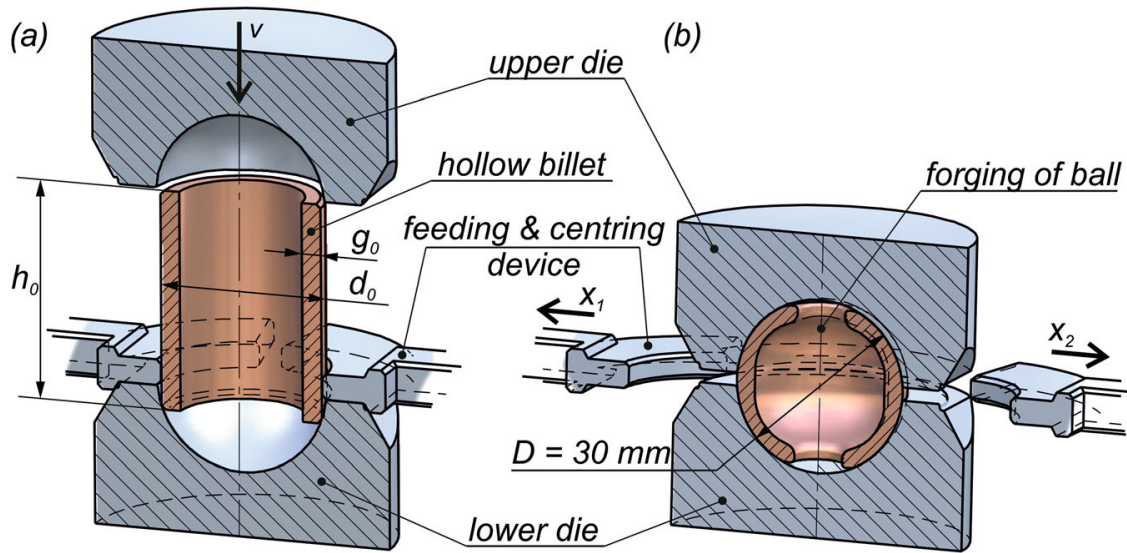

(c)

Fig. 3 A schematic of the process of forming a hollow ball forging $(a, b)$ and the tools used in the experiments (c)

simulation, it was determined that the gripper should be removed when the upper die moves about half the predetermined stroke. Delaying the moment of grippers retraction negatively inhibits a radial flow of the workpiece material. The upper die is forced against the billet to forge, in a single operation, a hollow ball with an outer diameter $D$. The forging has a wall of variable thickness and two coaxial openings (in its top and bottom parts) - shape-wise, the forging falls into the group shown in Fig. 2a. The dies are closed dies (i.e., without flash is expected to form), and their cavities have a spherical shape. The process is performed in a hydraulic press under cold forming conditions. The forging is blank with relatively small machining allowances which can be removed by grinding and, if need be, by finishing of the central holes (e.g., reaming or blanking), as proposed by Eklund and Campbell [22].

Figure $3 \mathrm{c}$ shows a photograph of the tools used in the laboratory tests. They consist of closed dies mounted in a device for coaxial movement. The stock material (billet shown in the figure) is fed and centered using a pair of grippers, whose design is simplified in comparison with the target industrial grippers. The tools were used to examine the actual forming conditions and to obtain information on the impact of the initial billet dimensions on the stability of the forging process.

\subsection{Scope of the study and methodology}

The focus of the present theoretical and experimental investigations is the process of forging a hollow ball with a nominal diameter $D=30 \mathrm{~mm}$ from tubular billets with initial wall thicknesses $g_{0}$ of $3 \mathrm{~mm}$ and $4 \mathrm{~mm}$. The experiments were conducted for a wide spectrum of combinations of initial billet heights and diameters. Based on preliminary tests, we decided that the experiments would be performed over an initial diameter $d_{0}$ range of 24 to $27 \mathrm{~mm}$ and an initial height $h_{0}$ range of 32 to $37 \mathrm{~mm}$. The combinations of dimensions $d_{0}$ and $h_{0}$ were selected in such a way as to obtain the same billet volume, which was predetermined separately for each individual wall thickness $g_{0}$. While selecting billet dimensions, we also took into account the values of slenderness ratios $h_{0} / d_{0}$ and $d_{0} / g_{0}$. Ultimately, the experiments were conducted using billets with $h_{0} / d_{0}$ ratios of $1.18-1.73$ and $d_{0} / g_{0}$ ratios of 5.75-9.00. These values excluded the possibility of global buckling of the billet during its shaping but unfortunately did not allow to eliminate local buckling (Alhussainy et al. [6]).

Experimental tests were carried out in laboratory conditions using the tools shown in Fig. $3 \mathrm{c}$ and an Instron tensile tester, which ensured constant die movement at a maximum speed of $1.67 \mathrm{~mm} / \mathrm{s}$ and a maximum force of $1 \mathrm{MN}$. During the experimental tests, tool movement was controlled by computer software, which additionally recorded the force and kinematic parameters. The working surface of the die cavity had been grease-lubricated each time before the billet was placed in the die (the composition of the lubricant is subject to trade secret). The workpiece was annealed commercial aluminum alloy AlMgSi0.5 (EN Al-6060-O according to EN 573-1, 2004). The tests were performed based on an incomplete block design, i.e., a method of experiments planning [24]. The range of the tested values of parameters was determined on the preliminary research. The limitations arising from the slenderness ratios were taken into account, too.

The theoretical analysis was performed using the Deform3D software, which is based on the finite element method (FEM). The scope of calculations was determined based on a complete block design.

In the calculations, it was assumed that the forging process was performed in cold conditions. Friction was described using the Coulomb model, assuming that the coefficient of friction between the forging and the tools was $\mu=0.24$, which corresponded to the experimental lubrication conditions. This value had been determined in a different study by the present authors (test method of upsetting ring workpieces and obtained results are described in detail in [25]).

The simulation assumed that the workpiece (billet) was formed in a hydraulic press in which the upper die moved at 
a constant speed of $v=1.67 \mathrm{~mm} / \mathrm{s}$. For obvious reasons, the aspects connected with the use of the feeding-centering device shown in Fig. 3a were ignored. Bearing in mind that the ball forging was made of $\mathrm{AlMgSi} 0.5$ alloy, calculations were done using a workpiece material model, which could be described by a constitutive equation of the following form [11, 25]:

$\sigma_{p}=949(0.0001+\phi)^{0.248} \exp (-0.5209 \phi) \dot{\phi}^{0.1358}$

where: $\sigma_{p}$, flow stress; $\varphi$, true strain; $\varphi$, strain rate. This model was determined based on the results of our previous research $[11,25]$. Plastometric tests were worked out using a strength machine. Cylindrical samples were upset at different values of strain rate. Finally, the constitutive equation (flow stress curves) describing the behavior of alloy was obtained with the author's program to a computer-aided experimental data analysis [25].

To minimize the calculation time and increase the accuracy of the results, in the simulation, we considered a case of spatial forming of a section of a billet (one-fourth of its perimeter), which was divided into approx. 200,000 tetragonal elements; there were at least 20 elements along the wall thickness. Symmetry boundary conditions were defined on two surfaces of this section. The scheme of the geometrical model is shown in Fig. 4. This figure shows a feeding-centering device, too. However, in the calculations, it was ultimately ignored. This tool was used only in the initial simulations, in order to estimate the time of retraction. The possibility of using a oneeighth section of the forging was also considered, but after initial calculations (Samołyk [2]) it was shown that this level of simplification was not recommended as it was accompanied by phenomena which destabilized the forming process. For a similar reason, an even simpler version of the simulation was abandoned, in which an axial-symmetric flat model was to be used.

\subsection{Forming a ball from a billet with a 3-mm-thick wall}

The reference ball, which was forged from a tubular billet with initial wall thickness $g_{0}$ of $3 \mathrm{~mm}$, diameter $d_{0}$ of $27 \mathrm{~mm}$, and height $h_{0}$ of $33.2 \mathrm{~mm}$, is shown in Fig. 5. The outer shape of the forging is quite characteristic (Fig. 5a). The central openings are encircled by two edges (an internal and an external one) between which a conical surface has formed. The state of this surface is basically the same as the surface of the nondeformed billet. Also, its width has not changed and is the same as initial thickness $g_{0}$, i.e., $3 \mathrm{~mm}$. The conical shape of this surface is due to the fact that the opening was formed by compressing the material, and the resulting outer edge moved, without much resistance, along the surface of the die cavity. Another characteristic area is the parting line zone. In the case of the forging under study, a cylindrical band (surface) with a width of approx. $2.5 \mathrm{~mm}$ was formed in this zone. Above and below this zone, there is a rounding zone. The remaining surface of the forging, which is the largest (percent-wise), has the expected spherical shape.

An analysis of an axial section of the reference forging (Fig. 5b) shows that it has a relatively regular shape. Admittedly, there are some local thickenings in which wall thickness ranges between 3.3 and $3.8 \mathrm{~mm}$, but we believe they are of little importance and the product is of good quality, especially considering the quality of the inner surface of the ball, which is de facto devoid of any technological defects. In addition, the forging has a shape similar to the theoretical one, shown earlier in Fig. 2a.

Figure 6a shows the most important dimensions of the axial section of the hollow ball (this is the reference forging shown in Fig. 5). The value of the outer diameter of the ball, depending on the measurement point, varies within a range of less than $3 \%$. Thus, assuming that a certain amount of stock is allowed for further machining, the investigated method makes it possible to obtain a perfectly spherical surface (after grinding) with a precisely defined outside diameter. Much larger variations in shape and dimensions were observed for the central openings. The values of the diameter measured within the inner edge can differ by as much as $12 \%$. In addition, it is not always possible to obtain a perfectly circular outline of this edge. On the other hand, assuming that the product is a semifinished one and that these holes will be subjected to further processing, the observed deviations should not pose a major technological problem. It should also be noted that the shape and dimensions of the central openings are the end product of the forming conditions. Figure $6 \mathrm{~b}$ shows the shape and dimensions of the forging calculated using FEM. In comparison with the experimental results, the forging obtained in the numerical simulation has a shape and dimensions closer to the expected ones. The outer surface is almost perfectly spherical and has a diameter equal to the pre-defined diameter of $30 \mathrm{~mm}$. In terms of quality and quantity, however, the differences between the finite element model and the ball obtained in the experiment are fully acceptable.

Changes in the shape of the wall of the investigated forging during the forming process and effective strain distribution are shown in Fig. 7. Initially, the billet is subjected to considerable compression, as an effect of which the central openings are formed. It is only later into the process (progress of over 60\%) that the billet clearly begins to upset, as a result of the increase in the contact surface between the material and the die cavity. During further stages of forming, one mainly observes an increase in the outside diameter of the workpiece in the plane of the parting line and a slower decrease in the diameter of the holes in the top and bottom part of the forging, with a more intense increase in wall thickness in the central zone of the ball. This is confirmed by the effective strain distribution. The highest strain values were observed in two zones, marked with the letters A and C (Fig. 7d). By contrast, zone B was 
Fig. 4 Scheme of the geometrical model of the forging process where the mesh quality is shown; description in text

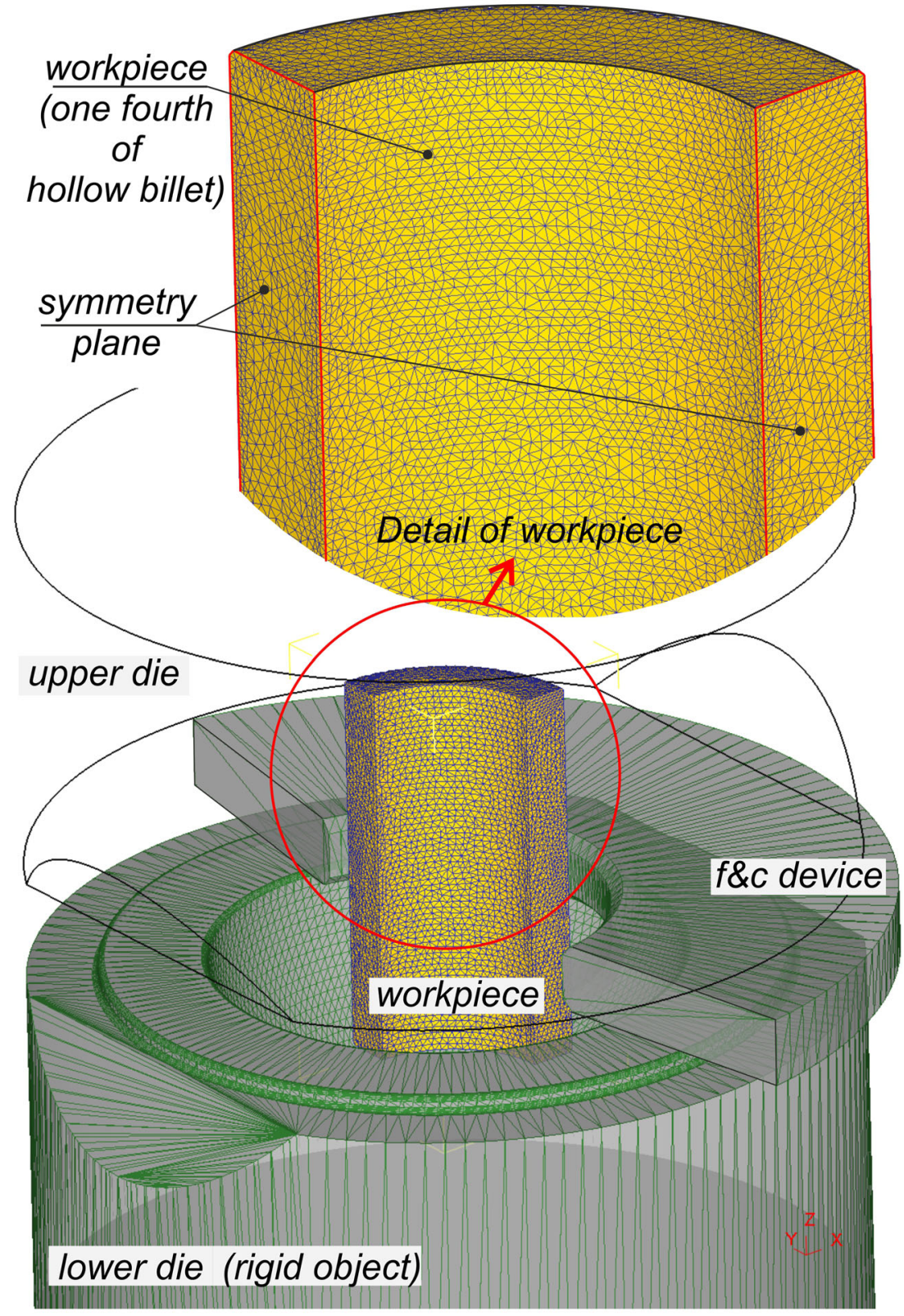

characterized by the lowest strain values, which, for most of the duration of the forming process, were close to zero. Such a distribution of this parameter may suggest that shape defects could occur on the outside of the forging around this zone (this issue will be discussed in greater detail for balls forged from a billet with a 4-mm-thick wall). These results also explain why the diameter of the central holes depends on the forming conditions. To obtain a forging with smaller openings, a billet with a greater initial height must be used, which, unfortunately, destabilizes the forming conditions.

Figure 8 shows the curve of forming force as a function of upper die stroke. This is the result of the reference ball forging. The experimentally determined force was compared with the force calculated using FEM. Generally, three phases of increase in the forming force can be distinguished. At first, the force grows slowly, in an almost proportional way. During this period, the billet is being compressed and the outer edges of the billet slide freely against the (inner) walls of the die cavity, resulting in the intensive formation of the central holes. It is only in the second stage, when flow resistance during compression increases, that the middle zone of the workpiece starts to be formed more intensively, which is represented by a marked increase in force in the force graph. In the final stage, the die cavities are fully closed upon the workpiece, which manifests as a very dramatic increase in force. This is typical behavior, often observed in closed die forging processes.

A comparison of the experimental results with FEM calculations demonstrates that the measured and calculated force 


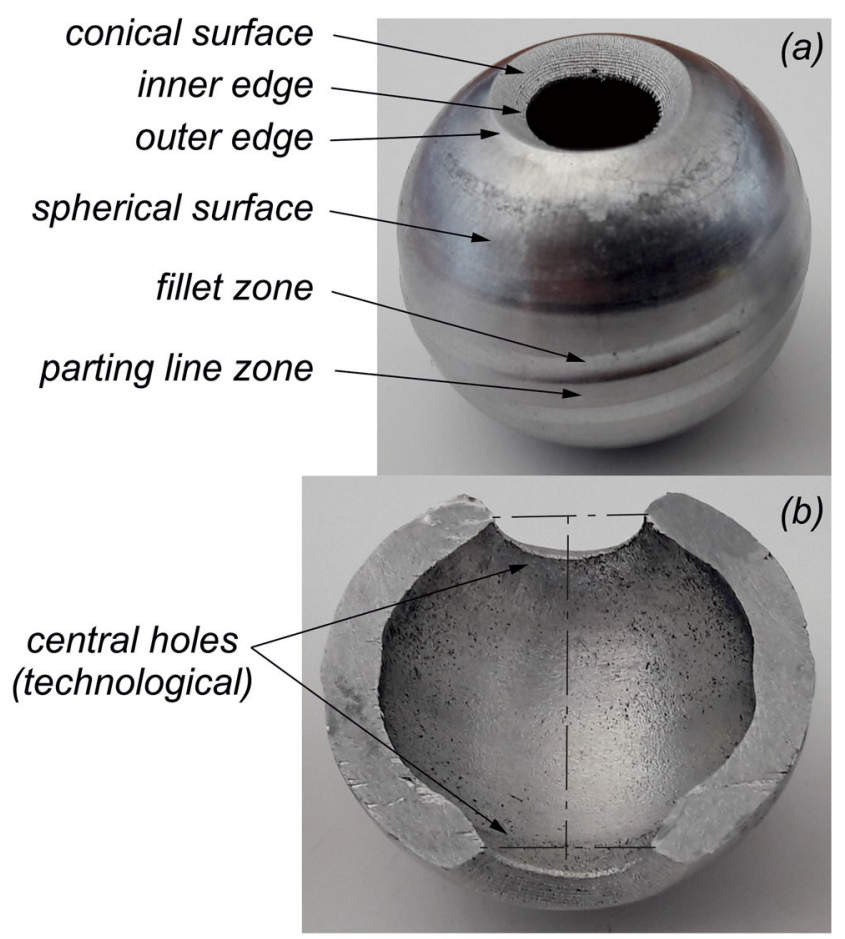

Fig. 5 Shape of a (reference) hollow ball forging obtained from a billet with diameter $\varnothing 27 \mathrm{~mm}$, wall thickness $g_{0}=3.0 \mathrm{~mm}$, and height $h_{0}=$ $33.2 \mathrm{~mm}$; description in the text

curves show very good agreement in the first stage of the forming process. Significant differences appear only in the second stage. During the simulation, the increase in the forming force is much greater than in real-life conditions. Nevertheless, the shapes of the curves are similar. Especially in the last stage of forging, both the calculated and the experimental force curves follow an identical growth trend. According to the authors' opinion, a friction condition has an influence upon the observed difference between the values of the force. A steady fiction is considered in numerical simulations; however, in reality, there is a variable friction. There can be observed that variability of friction conditions depends on the accuracy of making a die cavity, which has a spherical shape.

The experimental tests and numerical simulations demonstrate that the initial dimensions of the billet (and in particular the ratio between these dimensions) have a significant impact on the conditions of forming hollow ball forgings, thus, affecting the quality of the product or leading to shape defects. It turns out that slenderness ratio $h_{0} / d_{0}$ is an important parameter and should always be taken into account when designing the conditions for hollow ball forging. Unfortunately, there is only a very narrow range of slenderness ratio values favorable for forming hollow balls, which allow to obtain products of satisfactory quality. The discussed reference forging was forged from a billet with a $h_{0} / d_{0}$ ratio of 1.23 . A slight reduction in the value of this parameter, e.g., down to 1.21 , still ensures that the forging is of relatively good quality, and has a sufficiently

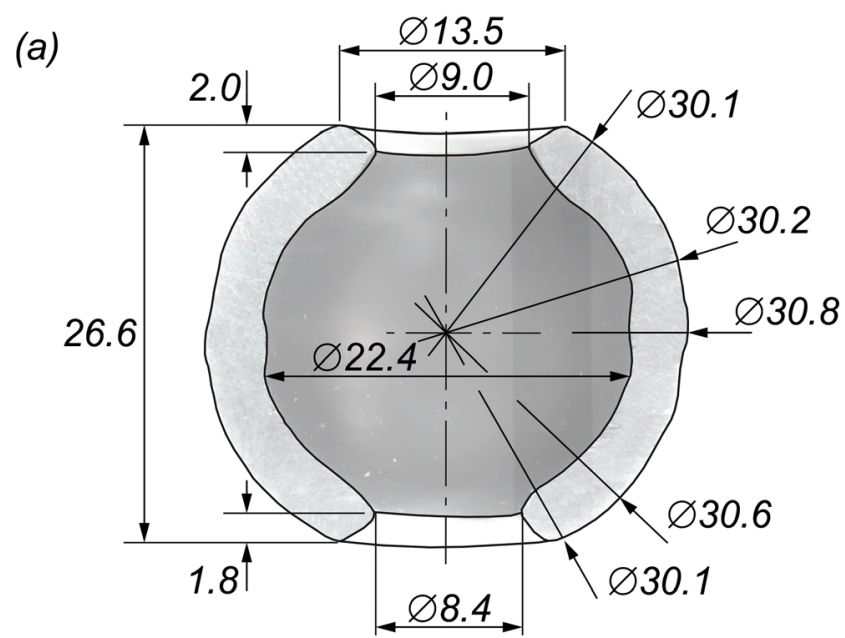

(b)

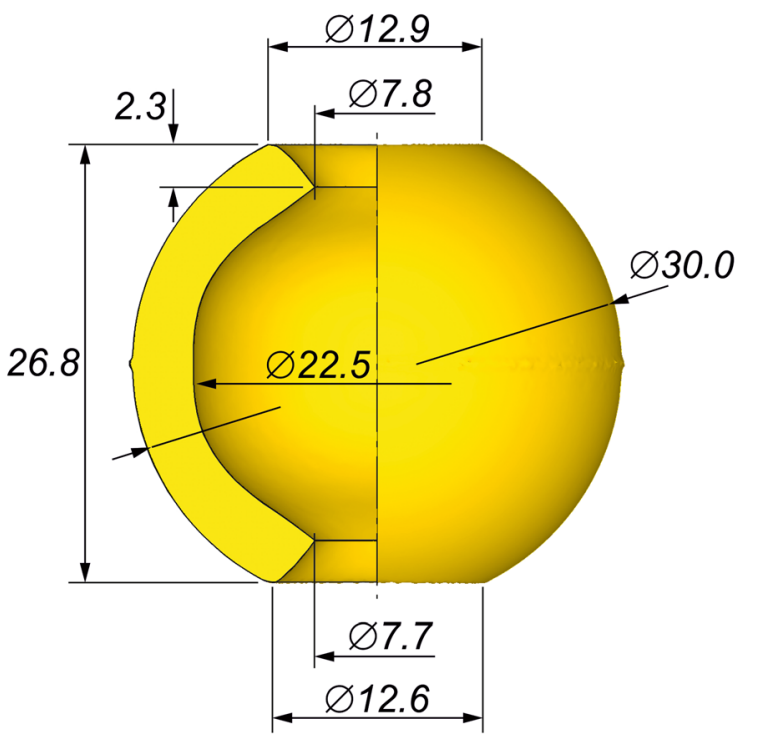

Fig. 6 Shape and the most important dimensions (in $\mathrm{mm}$ ) of the reference ball forging (forged from a billet with a wall thickness $g_{0}=3 \mathrm{~mm}$ ) obtained in the experiment (a) and by FEM calculations (b)

regular outer surface and central openings (Fig. 9a, b). However, at slenderness ratios lower than 1.20, the material starts to flow in between the dies (at the parting zone), creating an undesirable flash or deformation that resembles a flash line. Apart from the faulty shape of the forging, the use of a billet with an excessively low $h_{0} / d_{0}$ ratio results in a sharp increase in force which can lead to an overload of the forging machine and tools.

Yet, another undesirable effect that may appear during forming is local buckling that categorically disqualifies the forging. The experimental tests carried out in this study show that buckling occurs when the $h_{0} / d_{0}$ ratio is at least 1.33 (Fig. $9 \mathrm{c})$. It manifests as an unacceptable deformation of the forging wall in the plane of the parting line. The surface is concave, and at higher values of the slenderness ratio (Fig. 9d, at $h_{0} /$ $d_{0}=1.44$ ), it becomes strongly folded locally. An interesting fact is that the deformation of the forging wall is only observed 

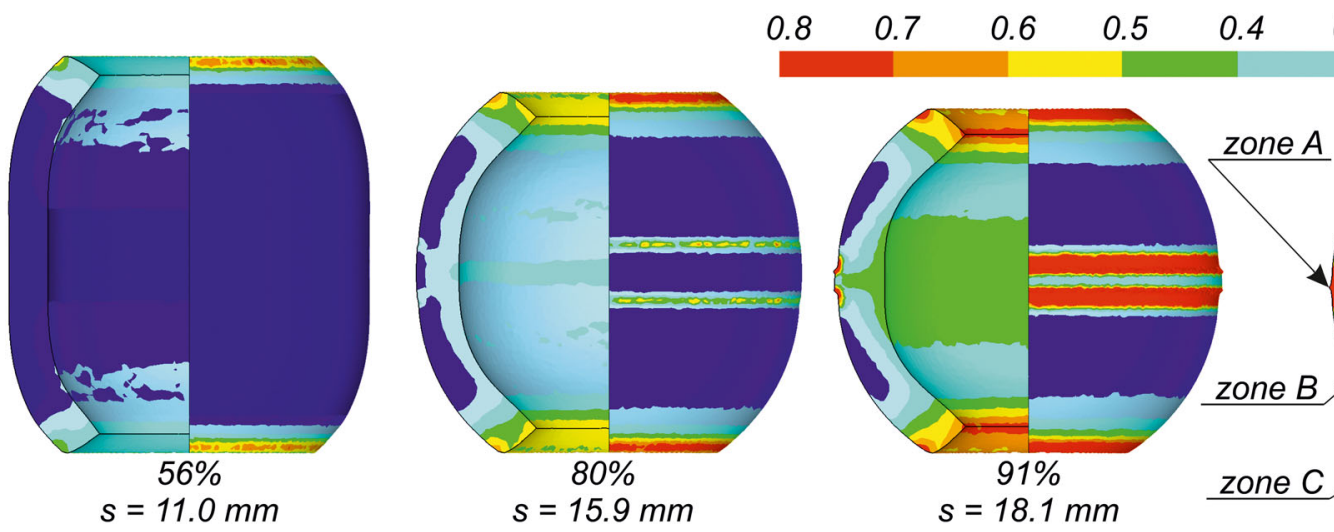

$0.3 \quad 0.2$

$0.1 \quad 0.0$

Fig. 7 Changes in the shape of the reference ball forging (obtained from a billet with wall thickness $g_{0}=3 \mathrm{~mm}$ ) calculated by FEM, and distribution of effective strain (where $s$ is the value of the stroke of the upper die)

along part of the perimeter, while in the simulation, buckling is observed along the whole perimeter of the forging. The FEM analysis, despite some incongruence with the actual conditions, shows that buckling can occur at $h_{0} / d_{0}$ ratio values that are only slightly higher than the limit values. FEM also allows to explain the mechanism of this phenomenon. We will return to this problem in another paper.

\subsection{Forming a ball from a billet with a 4-mm-thick wall}

Let us consider a process in which a hollow ball is forged from a billet with a higher initial wall thickness $g_{0}=4 \mathrm{~mm}$. The increase in wall thickness reduces the second slenderness

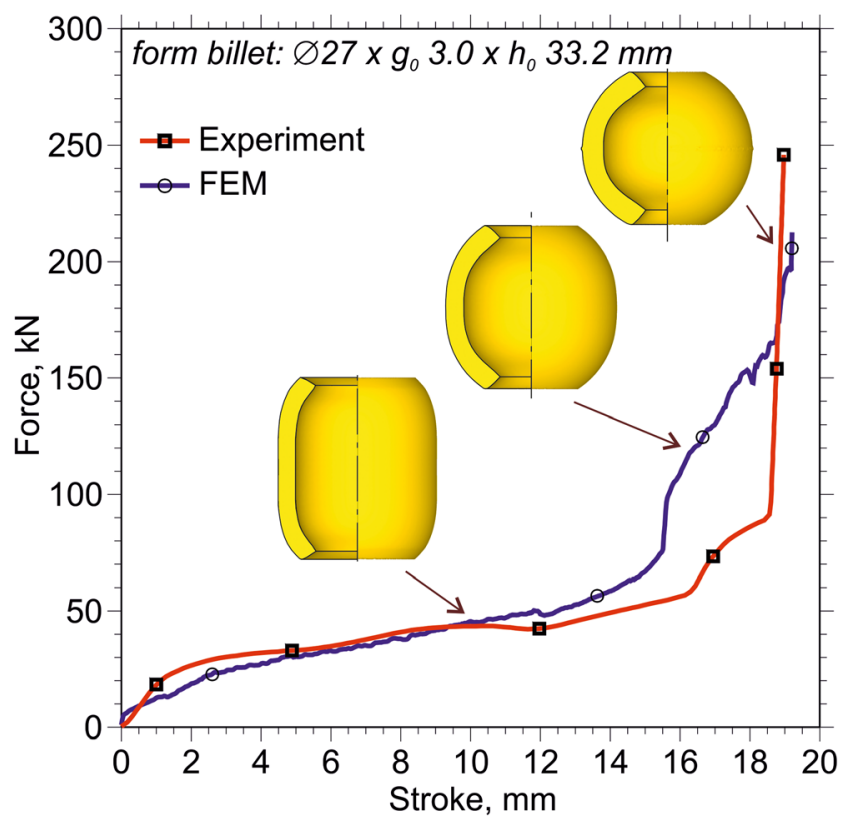

Fig. 8 Forming force curve calculated using FEM and measured in an experiment of forging a hollow ball from a billet with a wall thickness $g_{0}=3 \mathrm{~mm}$ (buckling) ratio $d_{0} / g_{0}$ compared with that of a billet with a 3mm-thick wall (from 7.5-9.0 to 5.5-6.5). In this part of the paper, we show that this slenderness ratio also substantially changes the conditions of forming a hollow ball forging, albeit not in the context of global buckling, as suggested by Alhussainy et al. [6].

A new reference forging (so-called) was used in this set of experiments; it was forged from a billet with initial wall thickness $g_{0}=4 \mathrm{~mm}$, diameter $d_{0}=25 \mathrm{~mm}$, and height $h_{0}=35 \mathrm{~mm}$. A view of this ball and its axial section are shown in Fig. 10. In comparison with the reference product discussed in the previous section (Fig. 5), the $h_{0} / d_{0}$ ratio increased from 1.23 to 1.40 , while the $d_{0} / g_{0}$ ratio dropped from 9.0 to as little as 6.25. This shows that increasing the wall thickness of a billet can considerably change forging conditions. Generally speaking, when a thick-walled billet is used, it is no longer as easy to produce a hollow ball forging of a fully satisfactory quality as it was in the previous case (see Fig. 5 or Fig. 9a, b), even though the typical limitations of the forging process are similar for both variants of the process. In the authors' opinion, this forging requires more finishing operations (by machining) to obtain the final product.

A comparison of the experimental results shown in Fig. 5 and Fig. 10 leads to the conclusion that the increase in initial wall thickness $g_{0}$ causes changes in several basic geometrical features of the forgings. Firstly, a frontal surface is formed between the two edges of the central opening, which is oriented perpendicular to the main axis of the forging (which passes through both central openings). At the same time, the outer edge becomes distinctly rounded. The changes in the geometry of this part of the forging are caused by an increase in flow resistance of the material (due to the change in $g_{0}$ ) and an increased effect of friction between the material and the walls of the die cavity. For the same reason, a furrow may form (it was observed only in some of the analyzed cases) between the two side surfaces of the forging - the spherical and the non- 


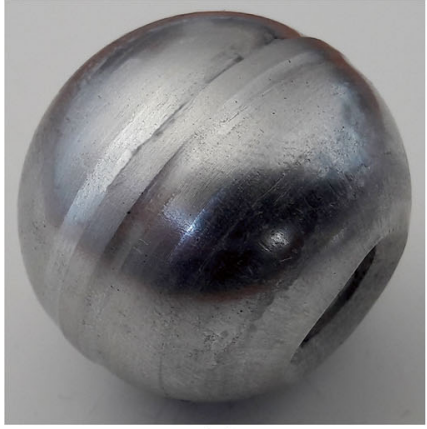

(a)

from billet:

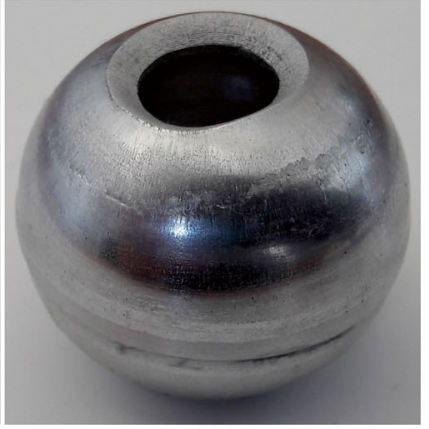

(b)

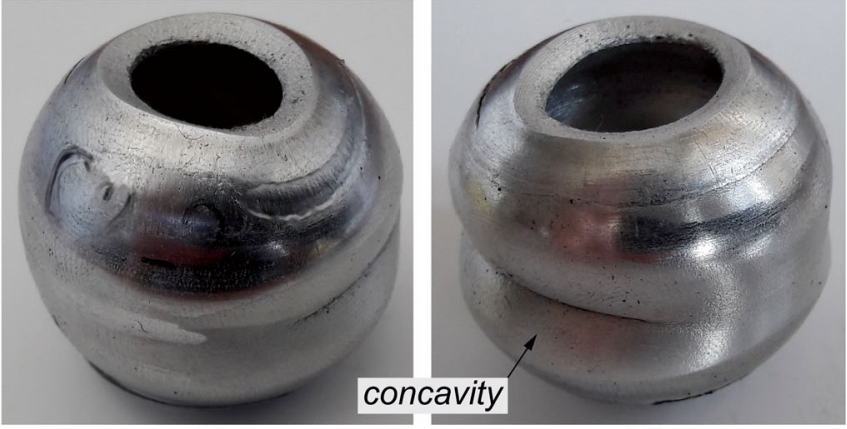

(c) from billet: from billet: from billet:

\section{the same as in case of (a) \\ $\varnothing 27 \times g_{0} 3.0 \times h_{0} 32.8 \mathrm{~mm}$ \\ $\varnothing 26 \times g_{0} 3.0 \times h_{0} 34.6 \mathrm{~mm}$ \\ $\varnothing 25 \times g_{0} 3.0 \times h_{0} 36.2 \mathrm{~mm}$}

Fig. 9 Examples of hollow ball forgings obtained from billets of various slenderness: 1.21

spherical surface. The latter type of surface is a defect (a limitation) that is characteristic of the discussed case of the forging process. It arises as a result of the less favorable material flow scheme compared with the one in which ball forgings are made from a billet with a thinner wall.

Increased flow resistance and greater impact of friction also contribute to the fact that the material has a greater tendency to move in the radial direction at the level of the parting line, which means the material flows in between the dies much earlier leading to the formation of flash (in some cases the flash was relatively large). One way to avoid this phenomenon is to reduce initial diameter $d_{0}$ of the billet, but this, unfortunately, is associated with the need to change height $h_{0}$; as a consequence, the forging is formed at less favorable slenderness ratios.

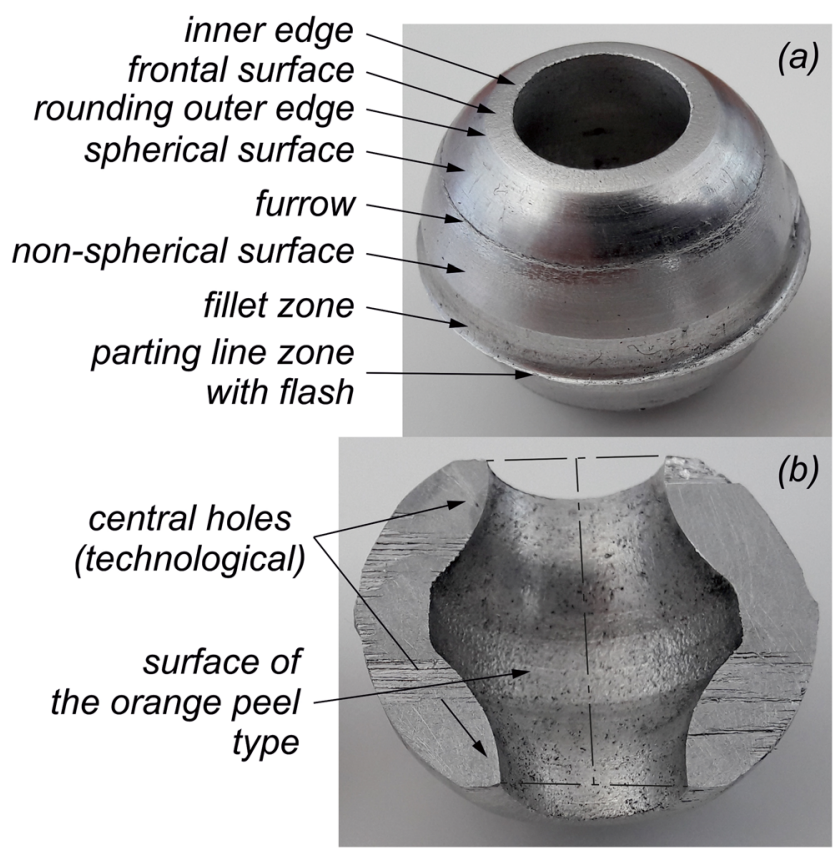

Fig. 10 View of a hollow ball forging obtained from a billet with diameter $\varnothing 25 \mathrm{~mm}$, wall thickness $g_{0}=4.0 \mathrm{~mm}$, and height $h_{0}=35 \mathrm{~mm}$; description in the text
It was observed for all analyzed dimensional variants of the billet that the coordinated action of increased flow resistance and friction during the forming of the hollow ball forging decreased the quality of its internal surface. This surface

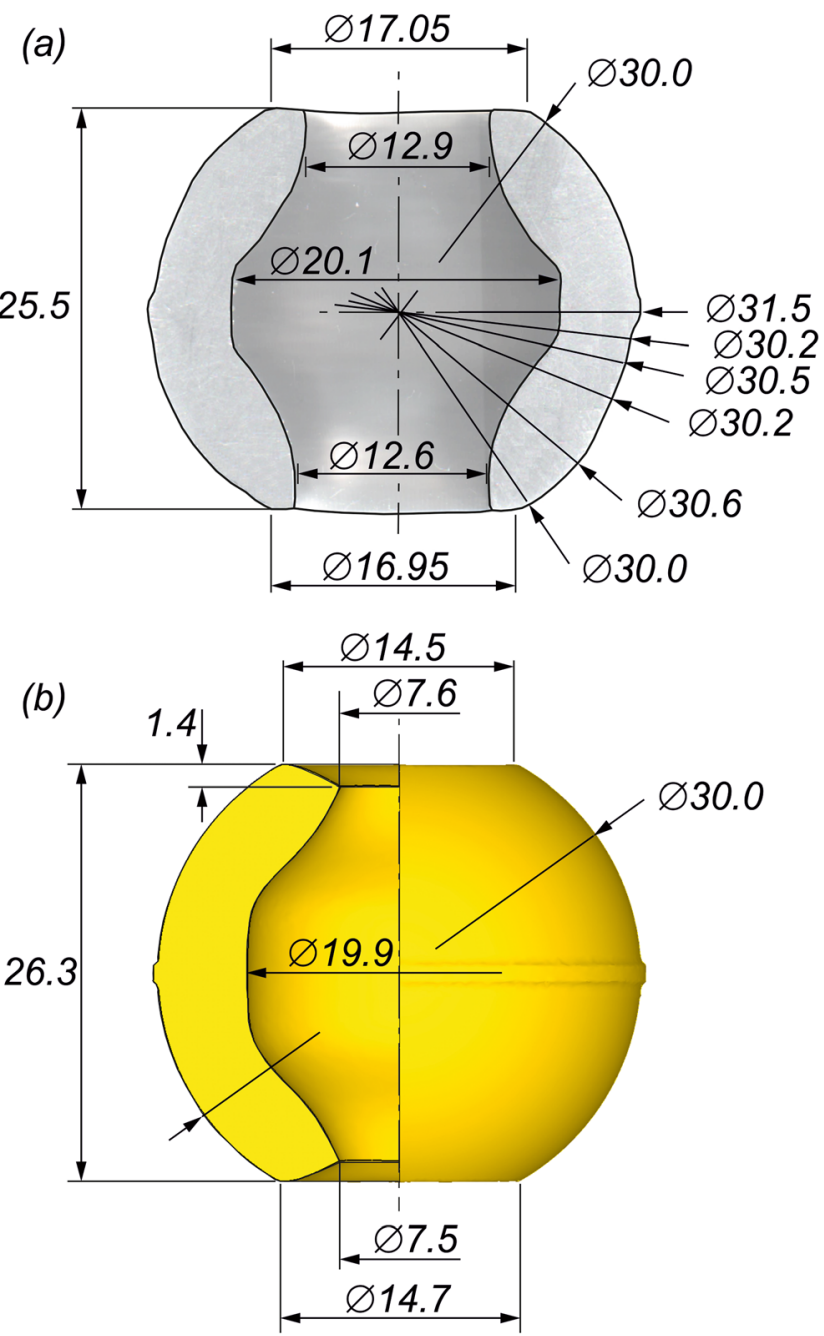

Fig. 11 Shape and the most important dimensions (in $\mathrm{mm}$ ) of a hollow ball forging formed from a billet with a wall thickness $g_{0}=4 \mathrm{~mm}$, obtained in the experiment (a) and by FEM calculations (b) 


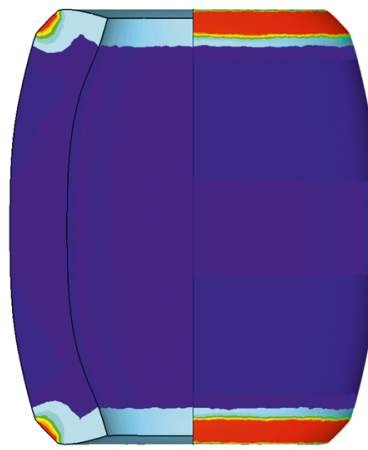

$46 \%$

$s=8.4 \mathrm{~mm}$

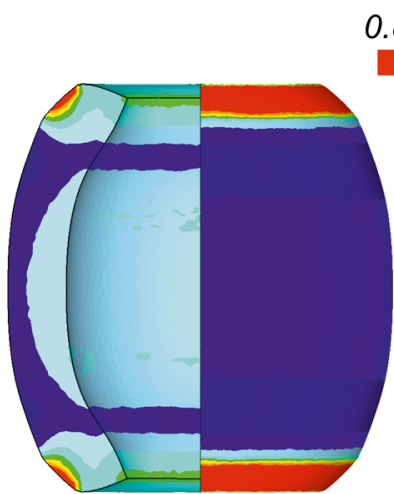

$65 \%$

$s=11.7 \mathrm{~mm}$

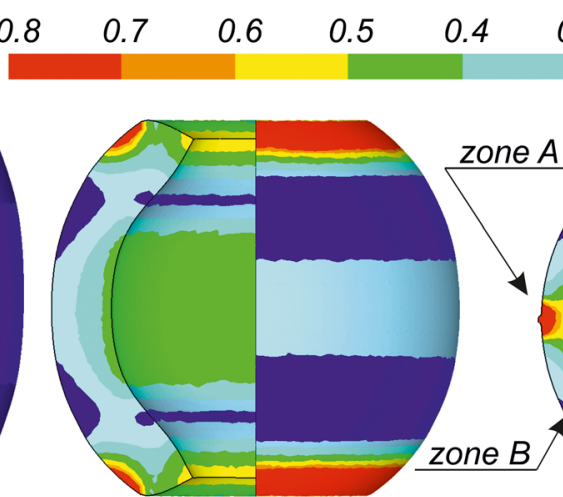

$84 \%$

$s=15.1 \mathrm{~mm}$

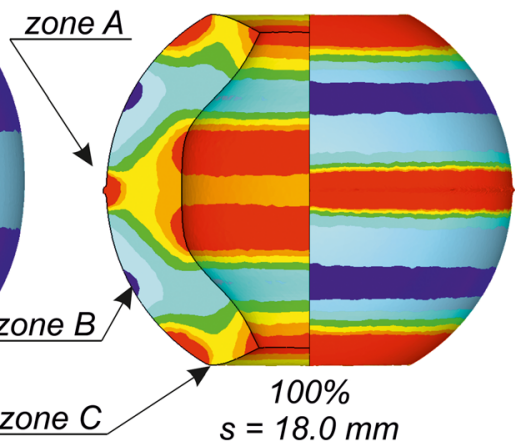

$\mathrm{s}=18.0 \mathrm{~mm}$

Fig. 12 Changes in the shape of the ball forging (obtained from a billet with wall thickness $g_{0}=4 \mathrm{~mm}$ ) calculated by FEM, and distribution of effective strain (where $s$ is the value of the stroke of the upper die)

(Fig. 10b) is visibly uneven, which also contributes to the irregular profile of the forging wall. A characteristic feature is the formation of a region in which surface defects may appear. Figure 10b shows a region like this with an "orange peel" surface. Additional information on the geometry of the obtained hollow ball forging is provided in Fig. 11, which shows the dimensions of the product. These dimensions were compared with the results of the numerical simulation. Unfortunately, the differences between FEM and the experiment are more pronounced here than for the ball forged from a thinner-walled billet. They mainly concern the shape around the central holes. Nevertheless, the results of FEM allow to explain certain aspects of this process. First of all, they confirm that the obtained shape of the forging near the holes is

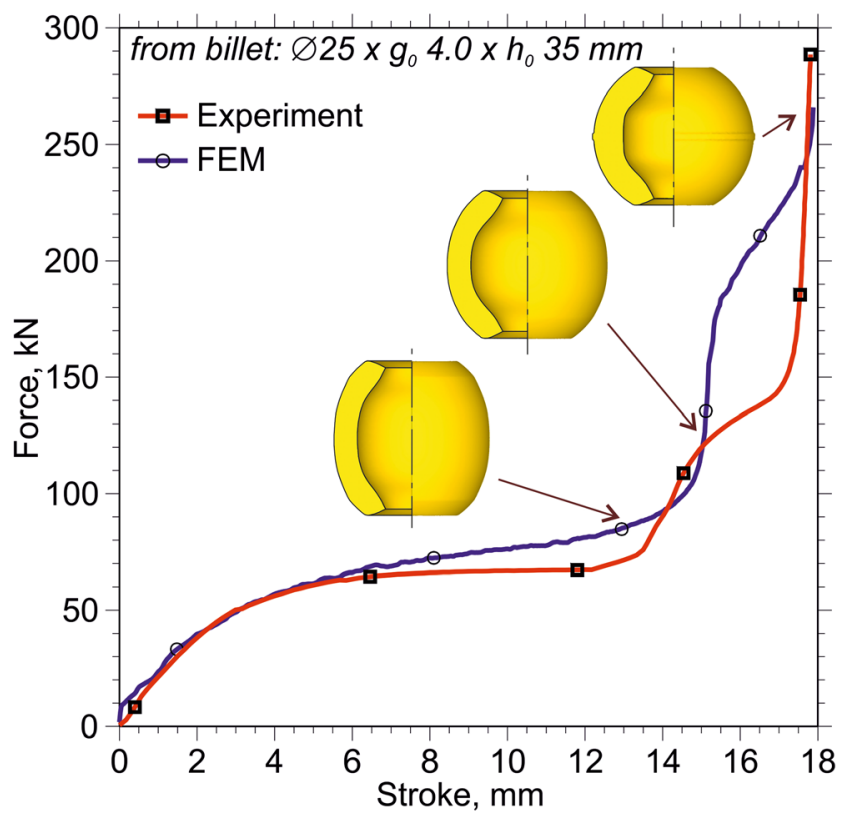

Fig. 13 Forming force curves calculated using FEM and measured in an experiment of forging a hollow ball from a billet with wall thickness $g_{0}=$ $4 \mathrm{~mm}$ substantially affected by friction between the material and the surface of the die. An analysis of the results of multivariate simulations demonstrates that the actual friction conditions are complex and it is extremely difficult (though not impossible) to reproduce them in a FEM model. In addition, it turns out that the use of the Coulomb model yields better results than, for example, the use of a constant friction model.

Figure 12 shows changes in the shape of the discussed hollow ball forging and effective strain distribution. By comparing the results of the calculations shown in Fig. 7 and Fig. 12, it can be demonstrated that while the differences in quality are negligible, quantitative differences are large. Particular attention should be paid to the three zones marked as A, B, and $\mathrm{C}$. The increase in the initial wall thickness of the billet increases strain in zones A and C. An analysis of effective strain distribution in zone $\mathrm{C}$ and the changes in the shape of the wall in this region of the forging (Fig. 12) confirm the earlier thesis about the increased friction, which is an indirect effect of the increase in material flow resistance and is directly related to the increase in initial wall thickness $g_{0}$. Strong friction of the outer edge of the workpiece against the surface of the die cavity changes the typical scheme in which the material around the central holes is being compressed, into a scheme in which radial flow of material towards the axis of the forging predominates.

The tendency to form the non-spherical outer surface mentioned above is explained by the low level of strain in zone B, which is additionally surrounded by an area characterized by a much higher effective strain. The way material flows in zone $\mathrm{C}$ has an indirect effect on the characteristics of zone $\mathrm{B}$. The FEM simulation shows that the outer surface of the forging in zone B does not come into contact with the surface of the die - the material "pulls off" from the cavity of the tool (this is confirmed by experimental results, Fig. 10a). To recapitulate, FEM results clearly demonstrate that large variations in effective strain distribution result in an irregular shape of the 


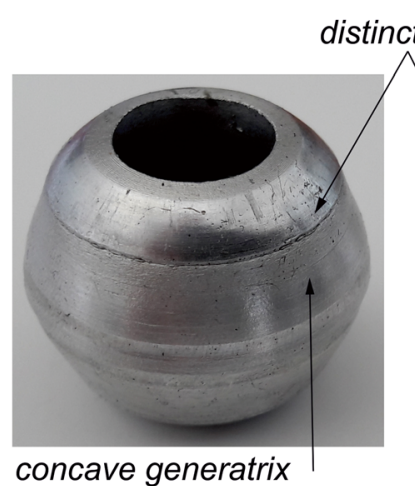

(a)

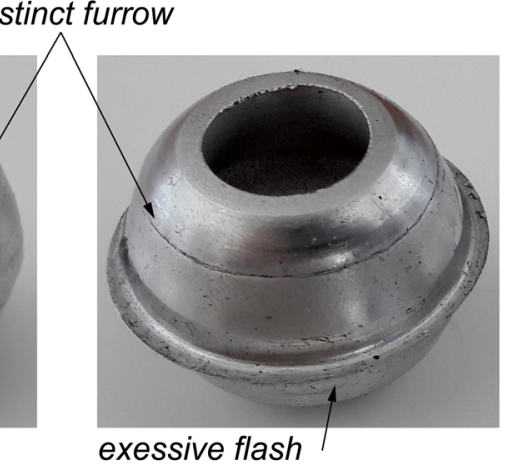

(b)

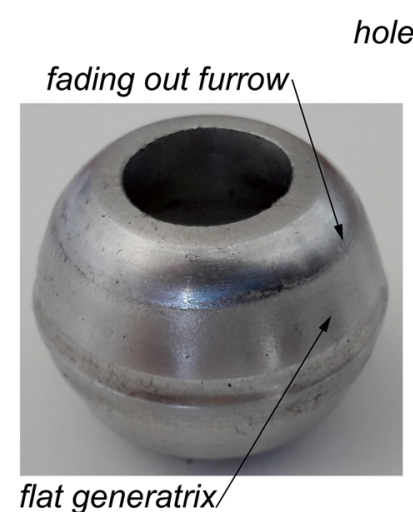

(c) hole with an irregular shape

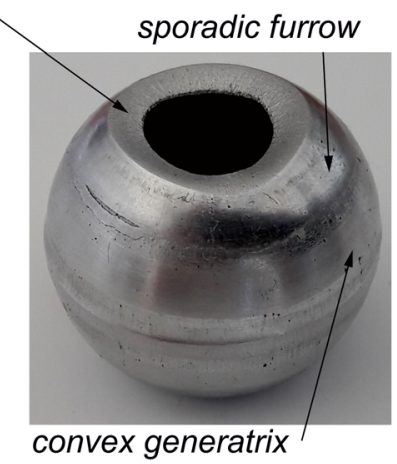

(d)

from billet:

from billet:

$\varnothing 25 \times g_{0} 4.0 \times h_{0} 33 \mathrm{~mm} \quad \varnothing 26 \times g_{0} 4.0 \times h_{0} 33.4 \mathrm{~mm}$

from billet:

$\varnothing 26 \times g_{0} 4.0 \times h_{0} 32 \mathrm{~mm}$

from billet:

$\varnothing 27 \times g_{0} 4.0 \times h_{0} 30 \mathrm{~mm}$

Fig. 14 Examples of hollow ball forgings obtained from billets of various slenderness: 1.32 (a), 1.28 (b), 1.23 (c), and 1.11 (d); description in the text

forging wall, and this is due to the increased flow resistance in zone $\mathrm{C}$.

Figure 13 shows forming force curves for the currently discussed hollow ball forging (presented in Figs. 10, 11, and 12), measured under experimental conditions and calculated using FEM. The observations are similar as in the case discussed in the previous section (Fig. 8). Initially, FEM predictions and experimental results show very good agreement. In the second half of the forming process, however, the differences between simulated and measured forces become quite pronounced. Nevertheless, qualitatively, the graphs are similar. Moreover, like in the case of Fig. 8, the forming process can be divided into three stages, with the reservation that when the ball is forged from a billet with 4-mm-thick walls, the increase in force at the beginning of each stage is more pronounced and abrupt.

It should be noted that the use of a billet with a smaller initial diameter $d_{0}$, even though it has a larger height $h_{0}$, shortens the forming path (Fig. 13), reducing thereby the forging time and the energy demand. This is the consequence of the shape of the die cavity, namely, the spherical shape causes changes in the forming path in a sinusoidal manner. However, in the light of the results presented above, it may turn out that the advantages of shortening the forming path by reducing the initial diameter of the billet may be outweighed by possible shape defects in the finished forging.

The final part of this section is devoted to the discussion of typical limitations of the process of forging a hollow ball from a tubular billet. When the ball was formed from a billet with a 4-mm-thick wall, no buckling or folding was observed, in contrast to the case shown in Fig. 9c, d (i.e., when $g_{0}=$ $3 \mathrm{~mm}$ ). However, a tendency for forming flash (Fig. 14b) and a defect in the shape of the outer surface (Fig. 14a) were observed. Flash formed primarily when the billet had a large initial diameter and an excessively large height (let us recall that height $h_{0}$ of the billet is increased to obtain forgings with the smallest possible central holes). To avoid the formation of flash, the height or diameter of the billet can be reduced; however, a change like this leads to defects in the side surface of the forging, as shown in the two selected cases in Fig. 14a and Fig. 14c. Nevertheless, it is possible to obtain a ball forging whose side surface will be of satisfactory quality. As shown in Fig. 14d, this can be achieved by using a billet with an initial diameter of $27 \mathrm{~mm}$ and a correspondingly reduced height $h_{0}$. Unfortunately, this is achieved at the cost of the quality of the central openings. To sum up, the technology of single-operation forging from a tubular billet does not allow to obtain a forging with a minimum diameter of central holes and a good quality outer surface.

\section{Conclusions}

In the paper, we discussed the results of experimental and theoretical studies of the process of the cold forging of a hollow ball from a tube-shaped billet. Two wall thicknesses of the billet were studied, which were chosen so that differences could be determined between the behavior of thinner and thicker-walled billets (i.e., with a 3-mm- and 4-mm-thick wall, respectively). The remaining initial dimensions of the billet, i.e., its height and diameter, were variable parameters. To generalize the obtained test results, the dimensions of the billet were additionally expressed as two dimensionless slenderness ratios. The following conclusions were formulated on the basis of the results obtained in the study:

- There is a very narrow range of values of billet dimensions at which an acceptable hollow ball forging can be obtained. 
- The obtained ball forging is characterized by an irregular wall thickness, and the degree of this irregularity depends on the initial wall thickness of the billet. The use of a tubular billet with a 4-mm-thick wall is associated with the appearance of defects on the inner surface of the forging, while the use of a billet with a 3-mm-thick wall increases the risk of local buckling.

- The two central holes formed during the process are an inherent part of the forging, and their diameter depends on processing conditions, in particular, the outer diameter and wall thickness of the billet.

- Use of a billet with a slenderness ratio $h_{0} / d_{0}$ higher than the limit value causes uncontrolled local buckling during the forming process, which leads to unacceptable shape defects, such as folding or non-spherical outer surface of the ball; the effect of buckling also depends on the wall thickness of the billet.

- When the slenderness ratio $h_{0} / d_{0}$ of the billet is too low, diameter $d_{0}$ has to be increased to a value that is too high in relation to the diameter of the ball $D$, which causes the formation of unwanted flash (can be of different size) in the plane of the parting line.

Open Access This article is distributed under the terms of the Creative Commons Attribution 4.0 International License (http:// creativecommons.org/licenses/by/4.0/), which permits unrestricted use, distribution, and reproduction in any medium, provided you give appropriate credit to the original author(s) and the source, provide a link to the Creative Commons license, and indicate if changes were made.

\section{References}

1. Zhang Y, Zhao S, Zhang Z (2008) Optimization for the forming process parameters of thin-walled valve shell. Thin-Walled Struct 46:371-379

2. Samołyk G (2018) Numerical analysis of a forging process for producing a hollow ball from tube. Adv Sci Technol Res J 12(3): $150-157$

3. Augustin C, Hungerbach W (2009) History and production of hollow spheres. Multifunctional Metallic Hollow Sphere Struct EM:530

4. Tomczak J, Pater Z, Bulzak T (2014) Designing of screw impressions in the helical rolling of balls. Arch Civ Mech Eng 14:104-113

5. Pater Z, Tomczak J, Bulzak T, Cyganek Z, Andrietti S, Barbelet M (2018) An innovative method for producing balls from scrap rail heads. Int J Adv Manuf Technol 97:893-901

6. Alhussainy F, Sheikh MN, Hadi MNS (2017) Behaviour of small diameter steel tubes under axial compression. Structures 11:155163
7. Pater Z, Tomczak J, Bulzak T (2016) An innovative method for forming balls from scrap rail heads. Adv Sci Technol Res J 10(31): $151-157$

8. Pater Z, Tomczak J (2012) Helical rolling of balls for ball mills. LUT Press, Lublin

9. Pater Z (2013) Multi-wedge cross rolling of balls. J Iron Steel Res Int 20(10):46-50

10. Cao Q, Hua L, Qian DS (2015) Finite element analysis of deformation characteristics in cold helical rolling of bearing steel-balls. J Cent South Univ 22(4):1175-1183

11. Samołyk G (2013) Studies on stress and strain state in cold orbital forging a AlMgSi alloy flange pin. Arch Metall Mater 58(4):11831189

12. Winiarski G (2013) Theoretical analysis of the forging process for producing hollow balls. Adv Sci Technol Res J 7(18):68-73

13. Kwan CT (2005) A study of process and die design for ball valve forming from stainless steel tube. Int J Adv Manuf Technol 26(910):983-990

14. Kozjek B, Seruga D, Popelnjak T (2017) Fatigue life prediction of brass ball forging tool. Mater Today-Proc 4(5):5855-5860

15. Kang JH, Lee HW (2016) Research on ball forging by ring rolling process. Int J Appl Eng Res 11(12):7823-7828

16. Skrochocki D, Tomczak J (2016) Numerical simulation of rotary compression process of hollow balls. Strength Mater 48(4):583591

17. Tomczak J, Pater Z, Bulzak T (2016) The influence of hollow billet thickness in rotary compression. Int J Adv Manuf Technol 82: 1281-1291

18. Berviller L, Bigot R, Martin P (2006) Technological information concerning the integrated design of "net-shape" forged parts. Int J Adv Manuf Technol 31(3-4):247-257

19. Chen CC (2015) Grain-size effect on the forging formability of mini gears. Int J Adv Manuf Technol 79(5):863-871

20. Wu P, Wang B, Lin J, Zuo B, Li Z, Zhou J (2017) Investigation on metal flow and forming load of bi-metal gear hot forging process. Int J Adv Manuf Technol 88:2835-2847

21. Eklund PE, Campbell GL (1973) Method of fabricating hollow balls for use in rolling contact bearing applications. US Patent 3774280A

22. Eklund PR, Campbell GL (1969) Method of making hollow balls for use in ball bearing and/or similar rolling operations. US Patent 3470720A

23. Braginsky M, Dashevsky L (1997) Process for the production of hollow ball bearings. US Patent 5659956A

24. Cox DR (1992) Planning of experiments. Wiley-Interscience Press, Hoboken

25. Samolyk G (2010) A flow stress model of formed 6000 series aluminum alloy in cold conditions. Ores and Non-ferrous Metals 55(6):326-329

Publisher's note Springer Nature remains neutral with regard to jurisdictional claims in published maps and institutional affiliations. 